\title{
TU/e EmonOWEN

\section{Time-resolved pinhole camera imaging and extreme ultraviolet spectrometry on a hollow cathode discharge in xenon}

\section{Citation for published version (APA):}

Kieft, E. R., Mullen, van der, J. J. A. M., Kroesen, G. M. W., \& Banine, V. Y. (2003). Time-resolved pinhole camera imaging and extreme ultraviolet spectrometry on a hollow cathode discharge in xenon. Physical Review E - Statistical, Nonlinear, and Soft Matter Physics, 68(5), 56403-1/10. [56403]. https://doi.org/10.1103/PhysRevE.68.056403

DOI:

10.1103/PhysRevE.68.056403

Document status and date:

Published: 01/01/2003

\section{Document Version:}

Publisher's PDF, also known as Version of Record (includes final page, issue and volume numbers)

\section{Please check the document version of this publication:}

- A submitted manuscript is the version of the article upon submission and before peer-review. There can be important differences between the submitted version and the official published version of record. People interested in the research are advised to contact the author for the final version of the publication, or visit the $\mathrm{DOI}$ to the publisher's website.

- The final author version and the galley proof are versions of the publication after peer review.

- The final published version features the final layout of the paper including the volume, issue and page numbers.

Link to publication

\section{General rights}

Copyright and moral rights for the publications made accessible in the public portal are retained by the authors and/or other copyright owners and it is a condition of accessing publications that users recognise and abide by the legal requirements associated with these rights.

- Users may download and print one copy of any publication from the public portal for the purpose of private study or research.

- You may not further distribute the material or use it for any profit-making activity or commercial gain

- You may freely distribute the URL identifying the publication in the public portal.

If the publication is distributed under the terms of Article 25fa of the Dutch Copyright Act, indicated by the "Taverne" license above, please follow below link for the End User Agreement:

www.tue.nl/taverne

Take down policy

If you believe that this document breaches copyright please contact us at:

openaccess@tue.nl

providing details and we will investigate your claim. 


\title{
Time-resolved pinhole camera imaging and extreme ultraviolet spectrometry on a hollow cathode discharge in xenon
}

\author{
E. R. Kieft, J. J. A. M. van der Mullen, * and G. M. W. Kroesen \\ Department of Applied Physics, Eindhoven University of Technology, P.O. Box 513, 5600 MB Eindhoven, The Netherlands \\ V. Banine \\ ASML Netherlands B.V., De Run 6501, 5504 DR Veldhoven, The Netherlands \\ (Received 16 June 2003; published 20 November 2003)
}

\begin{abstract}
A pinhole camera, an extreme ultraviolet (EUV) spectrometer, a fast gatable multichannel plate EUV detector, and a digital camera have been installed on the ASML EUV laboratory setup to perform time-resolved pinhole imaging and EUV spectroscopy on a copy of the Philips EUV hollow cathode discharge plasma source. The main properties of the setup have been characterized. Time-resolved measurements within the plasma pulse in the EUV have been performed on this source. Specific features of the plasma, such as a ring shape in the initiation phase and a propagating sphere during the pinch phase, have either been discovered or confirmed experimentally. Relative populations of various ionization stages in the pinch plasma have been estimated on the basis of line intensities and calculated transition probabilities. The changes in relative line intensities of a single ionization stage can be explained by a combination of temperature and excitation/deexcitation balance effects. Experiments with argon dilution on a newer version of the source show considerable effect on the shape of the xenon EUV spectrum.
\end{abstract}

DOI: 10.1103/PhysRevE.68.056403

PACS number(s): 52.25.Os, 52.70.La, 52.80.-s

\section{INTRODUCTION}

\section{A. Background}

Various concepts of discharge plasmas are currently under development by a number of groups worldwide as candidate sources of extreme ultraviolet (EUV) radiation for application in future lithography tools (for a recent overview, see, e.g., Refs. [1,2]). Common properties of these plasmas are that they have small spatial dimensions (typically less than 1 $\mathrm{mm}$ ) and occur in short pulses (of the order of $100 \mathrm{~ns}-1 \mu \mathrm{s}$ duration) rather than as steady-state discharges.

To gain insight in the characteristics of such discharges, it is necessary to develop and apply diagnostics that not only show the required spatial resolving power but also operate on time scales shorter than the typical pulse duration.

\section{B. Basic description of the Philips source}

In the ASML EUV laboratory in Veldhoven, The Netherlands, an early state-of-development version copy of the Philips EUV hollow cathode discharge plasma source was installed in an experimental setup in the end of 2001. The concept of this EUV source was described first in Refs. [3-5]. See Fig. 1 for a schematic representation of the source. Initially, the total capacitance of the electrical circuit for this specific source was $48 \mathrm{nF}$. The source was operated with voltages up to $15 \mathrm{kV}$. This version was used as a source of photons for testing purposes (especially of mirror lifetime during exposure to the plasma) and as a study object for the initial characterization of the pinch plasma.

The source received intermediate updates from the supplier, including an adjustment of the electrode geometry and

\footnotetext{
*Corresponding author. Email address: j.j.a.m.v.d.mullen@tue.nl
}

a change in the electrical parameters towards a higher capacitance of $674 \mathrm{nF}$ and lower working voltage of $3 \mathrm{kV}$ at maximum. All experiments described here, except for those concerning dilution of the working gas with argon, have been performed on the "basic" version of the source.

\section{This work}

In the framework of a joint research project between ASML and TU/e, involving both theoretical and modeling efforts and experimental investigations, fast EUV imaging and fast spectroscopy on this source have been combined to further the knowledge on the dynamics of the EUV plasma pulse. The combination of time-resolved EUV imaging and spectroscopy has led to better understanding of the dynamics

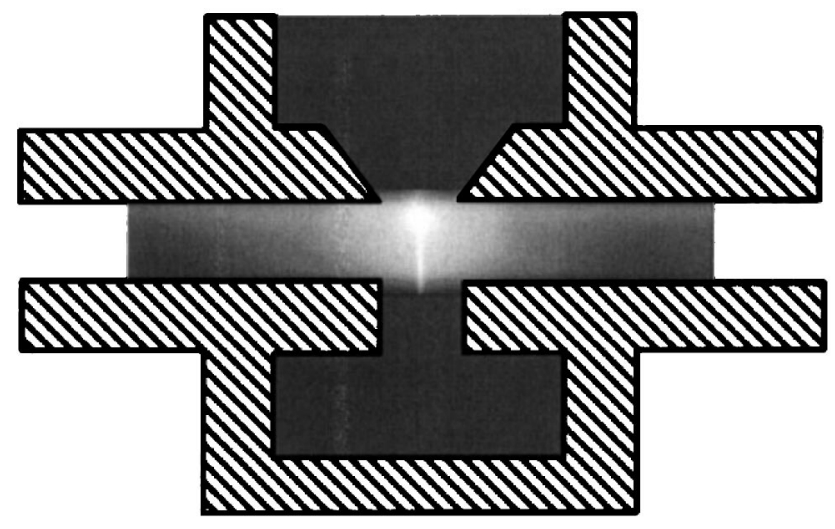

FIG. 1. A schematic cross-section representation of the hollow cathode EUV source, reproduced from Ref. [3]. The anode is at the top and the hollow cathode at the bottom. The pinched plasma is represented by the bright spot in the center. The source is cylinder symmetrical; EUV radiation is normally collected through the hole in the anode. 


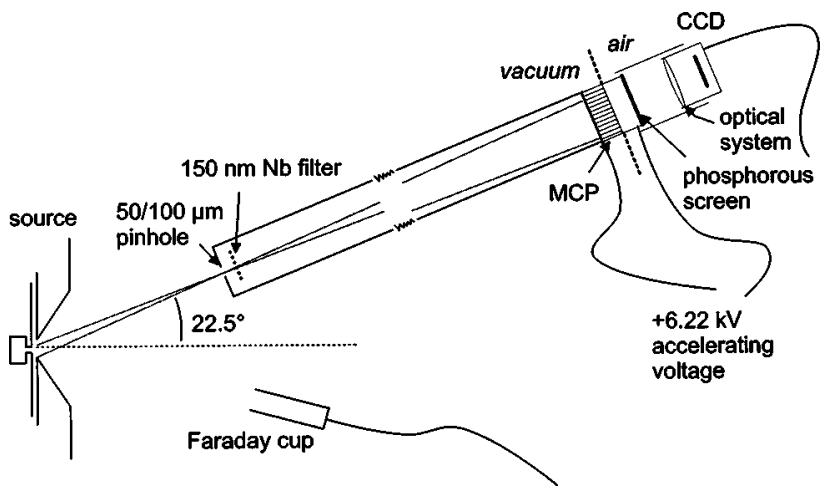

FIG. 2. A schematic view of the pinhole camera setup.

of the pinch plasma in the Philips EUV source. Owing to good reproducibility in timing of both the plasma pulse and the trigger signal, a time resolution of $10 \mathrm{~ns}$ or better could be achieved while integrating light from several pulses, making it possible to resolve the different phases of the plasma during the discharge pulse. Abel inversion was applied to space-resolved EUV spectrometry to plot the EUV line intensities during a ring-shaped phase of the discharge. Calculations based on the COWAN computer code [6] were used to derive rough estimates of the relative populations of different ionization stages of xenon in the plasma.

\section{EXPERIMENT}

For typical experiments as described here, the source was operated in a repetitive mode at $100 \mathrm{~Hz}$ repetition frequency. The dead time of the high-voltage supply for the multichannel plate (MCP) camera limited the recordings of pinhole images or EUV spectra to just one pulse per second. Several pulses (typically 10 or 30) were integrated for each image to gather sufficient intensity and reduce the effect of short time scale variations in the plasma characteristics.

\section{A. Pinhole camera}

A pinhole camera, consisting of a pinhole, a filter, a MCP image intensifier from the ISAN institute in Troitsk, Russia [7], and a commercial-type digital camera, was applied to record time-resolved images of the discharge plasma in the EUV wavelength range. For low power applications, the system provides a simple and cost-efficient imaging alternative to mirror projection systems. Images were recorded with the camera in two different positions: one on the axis of symmetry of the source and one at an angle of $22.5^{\circ}$ with respect to that axis. A schematic representation of the setup is shown in Fig. 2.

Just behind a (electrical spark or mechanically drilled) 50or 100- $\mu$ m-diameter pinhole, a 150-nm-thick niobium/ silicon filter was positioned. It has a transparency of about $50 \%$ to EUV light with a wavelength between roughly 10 and $17 \mathrm{~nm}$, but it blocks over $99 \%$ of any vacuum ultraviolet (VUV) light emitted by the source at larger wavelengths. The supporting mesh of the filter has $0.5-\mathrm{mm}$ spacing and is visible in some of the pinhole images. The pinhole image is projected onto the surface of a MCP with phosphorus screen, which intensifies the signal and converts the EUV radiation into visible light. The MCP was gated by a fast $6.25-\mathrm{kV}$ high-voltage pulse over the plate and the adjacent gap to the phosphorus screen. The typical duration of the pulse was 10 ns. However, variation of the pulse duration showed that the effective exposure time was only $5 \mathrm{~ns}$, a fact that is probably caused by capacitive properties of the current circuit. The phosphorus screen also served as a vacuum-to-air interface, and the intensified image was finally recorded by a highsensitivity commercial digital camera.

Depending on the distance between the plasma and the pinhole, and pinhole diameter, the spatial resolution in the plasma that could be achieved was in the order of $70 \mu \mathrm{m}$. The two main contributors to this resolution are a geometrical contribution and diffraction from the pinhole at the EUV wavelengths. Serious blurring of the image due to diffraction at larger wavelengths was prevented by the niobium/silicon filter and the drop of sensitivity of the MCP above $100 \mathrm{~nm}$ [8].

The distance from the pinhole to the MCP surface was fixed at $770 \mathrm{~mm}$; the distance from the source to the pinhole was $140 \mathrm{~mm}$ for the off-axis position and $130 \mathrm{~mm}$ for the on-axis position, resulting in magnification factors from source to MCP of 5.5 and 5.9, respectively.

\section{B. EUV/VUV spectrometer}

For the EUV spectrometer, partly the same arrangement was used as for the pinhole camera. A $100-\mu \mathrm{m}$ slit was mounted at the position of the MCP camera. A $12001 / \mathrm{mm}$ grazing-incidence reflective grating was used as the dispersing element to record the EUV spectrum from roughly 9 to $18 \mathrm{~nm}$. A different grating with $300 \mathrm{1} / \mathrm{mm}$ could be fitted to cover the VUV range from roughly 30 to $90 \mathrm{~nm}$. However, since the higher ionization stages of xenon $(7+$ and above) all radiate in the EUV, and interpretation of the EUV part of the spectrum turned out to be relatively simple, only spectra recorded with the $1200 \mathrm{l} / \mathrm{mm}$ grating are discussed in the present work.

A valve with a bypass tube was used to prevent degradation of the optical elements during operation of the source without using the spectrometer, while keeping the spectrometer at vacuum pressure. Again, the MCP and digital camera were used for intensification, conversion, and recording of the spectrum. The MCP was mounted in an off-Rowland circle configuration for simplification of alignment and mounting.

Both gratings of the spectrometer have $1000 \mathrm{~mm}$ radius of curvature. The grazing angle of incidence is $4^{\circ}$ and the center of the MCP is located at a grazing angle of $10^{\circ}$ from the grating. The slit is on the Rowland circle of the grating. The MCP is mounted onto a holder with adjustable distance to the grating, so that the wavelength for which it is "focused" onto the Rowland circle can be adjusted. A spectral resolution of $\Delta \lambda=0.1 \mathrm{~nm}$ or better can be achieved with this setup.

The background pressure of xenon gas along the path between the pinhole position and the MCP was kept below 0.1 $\mathrm{Pa}$ to minimize reabsorption of the EUV radiation. For this 


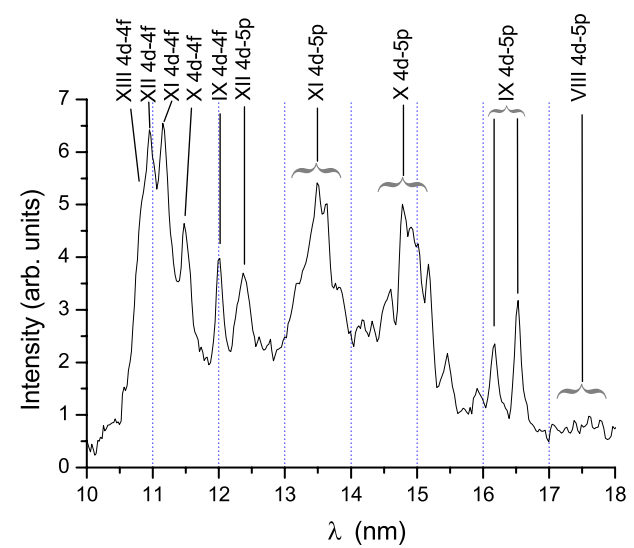

FIG. 3. Sample EUV spectrum recorded using a 1200 1/mm grazing-incidence grating spectrograph with MCP.

pressure, the absorption in Xe in the EUV range over $1 \mathrm{~m}$ path length is $6 \%$ at most. A $30001 / \mathrm{s}$ turbo pump was used to pump off the xenon flow of about 8 SCCM (cubic centimeter per minute at STP) that was required to run the discharge. In the experiments with argon dilution, an argon gas buffer was applied in combination with a downstream flow restriction (not obstructing the view to the pinhole camera or EUV spectrometer). In this case, the total gas flow was much larger; however, due to the much lower absorption cross section for argon, the contribution of argon to the absorption of EUV radiation is negligible.

To obtain spatially resolved spectra from the on-axis position, an additional slit was inserted at the pinhole position and orientated perpendicular to the spectrometer entrance slit. The spatial resolution of the spectra is comparable to that of the pinhole images. Since the source electrodes have cylindrical symmetry, Abel inversion has been applied to obtain line intensities in the plasma as a function of the distance to the symmetry axis.

An example of the EUV spectrum of xenon in this plasma, with classification of its main features, is shown in
Fig. 3. The identifications are supported by literature [9-11] and/or calculations based on the COWAN computer code [6]. Some difficulty arises with the lines around and below 11 $\mathrm{nm}$, since many lines from stage XII and higher tend to overlap here. The contribution from stage XIII was derived from a distinctly different behavior of the intensity at $10.8 \mathrm{~nm}$ compared to that of larger wavelengths, during variation of the plasma parameters. Since the contribution had to come from one or more stages higher than XII, and stages XIV and higher are unlikely to be populated under the given plasma settings, it was ascribed to Xe XIII.

For evidence of the presence of different ionization stages at a certain place or time in the plasma, the $4 d-5 p$ transition features have been used wherever possible because of the fact that they are well separated for the different stages. However, in the initiation phase of the discharge, the intensities of these features are very low, and the stronger $4 d-4 f$ transitions had to be applied for identification of the stages. For an overview of the EUV lines used for identification of the ionization stages, see Table I.

\section{Triggering and other metrology}

As stated above, fast and reproducible gating of the imaging system was achieved by applying short high-voltage pulses to the MCP camera. Given the unavoidable delays caused by the application of the high-voltage pulse generator, a Stanford DG535 delay generator, and some lengths of coaxial cables, a sufficiently strong and low-jitter trigger signal was needed to be available at least $160 \mathrm{~ns}$ before the onset of any EUV radiation from the discharge. Since the source operated in a "self-breakdown" mode, no such signal was available from the control electronics of the source.

However, it is a common characteristic of hollow cathode discharges, known both from experiments and modeling (see, e.g., Refs. $[12,13]$ ), that they emit an electron beam before the discharge in the main electrode gap. Such an electron beam produces a negative voltage on a Faraday-cuptype collector that is placed close to the axis behind the anode hole. A sufficiently strong signal with a jitter of no more than $\sim 2$ ns could be picked up on-axis at least 300 ns before

TABLE I. An overview of the EUV lines in the spectrum of the discharge used for ion identification. The listed intensities refer to typical maximum measured intensities of the line during the discharge, and serve only for relative comparison within one part of the spectrum. For Xe $\mathrm{x}$, the weighted transition probability (gA) values are those of the strongest transition at the given wavelength.

\begin{tabular}{ccccccc}
\hline \hline$\lambda_{\text {obs }}(\mathrm{nm})$ & Stage & Lower level & Upper level & $\mathrm{gA}\left(10^{9} \mathrm{~s}^{-1}\right)$ & $I$ (counts) & Reference \\
\hline 17.77 & VIII & $4 d^{10} 5 s$ & $4 d^{9} 5 s 5 p$ & & 2000 & {$[10]$} \\
12.01 & IX & $4 d^{10}{ }^{1} S_{0}$ & $4 d^{9} 4 f{ }^{1} P_{1}$ & 4554 & 6500 & {$[9]$} \\
16.53 & IX & $4 d^{10}{ }^{1} S_{0}$ & $4 d^{9} 5 p{ }^{1} P_{1}$ & 241 & 6000 & {$[9]$} \\
11.49 & X & $4 d^{9}$ & $4 d^{8} 4 f$ & 14400 & 5500 & {$[9]$} \\
14.80 & X & $4 d^{9}$ & $4 d^{8} 5 p$ & 321 & 5500 & {$[9]$} \\
11.15 & XI & $4 d^{8}$ & $4 d^{7} 4 f$ & & 5000 & {$[11]$} \\
13.52 & XI & $4 d^{8}$ & $4 d^{7} 5 p$ & & 3500 & {$[6,11]$} \\
10.96 & XII & $4 d^{7}$ & $4 d^{6} 4 f$ & & 2500 & {$[11]$} \\
12.39 & XII & $4 d^{7}$ & $4 d^{6} 5 p$ & & 3500 & {$[6]$} \\
10.80 & XIII & $4 d^{6}$ & $4 d^{5} 4 f$ & & & {$[6]$} \\
\hline \hline
\end{tabular}


the main discharge. In the present setup, the cup was placed at a slightly off-axis position to allow space for the pinhole camera. Still, a sufficiently strong signal was picked up in time for recording even the earliest phases of EUV emission in the discharge.

The same Faraday cup signal can also be used to detect the arrival of ions from the discharge plasma expansion. From the time delays between the plasma pinch and the arrival at the Faraday cup, the ion velocity distribution can be derived.

A copy of the so-called "Flying Circus" tool [14], consisting of a set of photodiodes behind near-normal incidence multilayer mirrors, was used to measure the absolute in-band EUV output of the source to have a simple measure of the proper operation of the source, and its stability.

\section{Derivation of relative ionization stage populations}

Relative population densities of the different xenon ionization stages have been estimated by comparison of the measured EUV spectra with theoretical calculations of the positions and transition probabilities of xenon lines in the EUV. The theoretical data were provided by calculations using the COWAN computer code [6]. The spectral resolution of the spectrometer was about $0.1 \mathrm{~nm}$; therefore, lines within a range of $0.1 \mathrm{~nm}$ in total were taken to contribute to the measured intensity at a certain wavelength.

Furthermore, local thermodynamic equilibrium (LTE) and a fixed electron temperature $T_{e}=35 \mathrm{eV}$ have been assumed to relate line intensities to the ground state population densities. In short, the relative population densities were calculated as

$$
n_{Z}=\frac{n_{Z}^{\prime}}{\sum n_{i}^{\prime}},
$$

with

$$
n_{Z}^{\prime}=\frac{I_{\text {meas }}}{\sum g_{n} A_{n m}} \exp \left(-\Delta E / k T_{e}\right)
$$

where the first summation is over all considered ionization stages and is carried out for normalization purposes. The second summation goes over the relevant transition lines from the computer code output as discussed above.

The assumption of LTE is necessary for this derivation. However, since all the applied lines are related to similar transitions and lie relatively close together in terms of wavelength and energy levels, it is likely that any deviations from LTE will affect the intensities of the different lines in similar ways. Therefore, these calculated relative population densities may also serve as rough estimates of the densities in the non-LTE conditions that are encountered in different phases of the discharge.

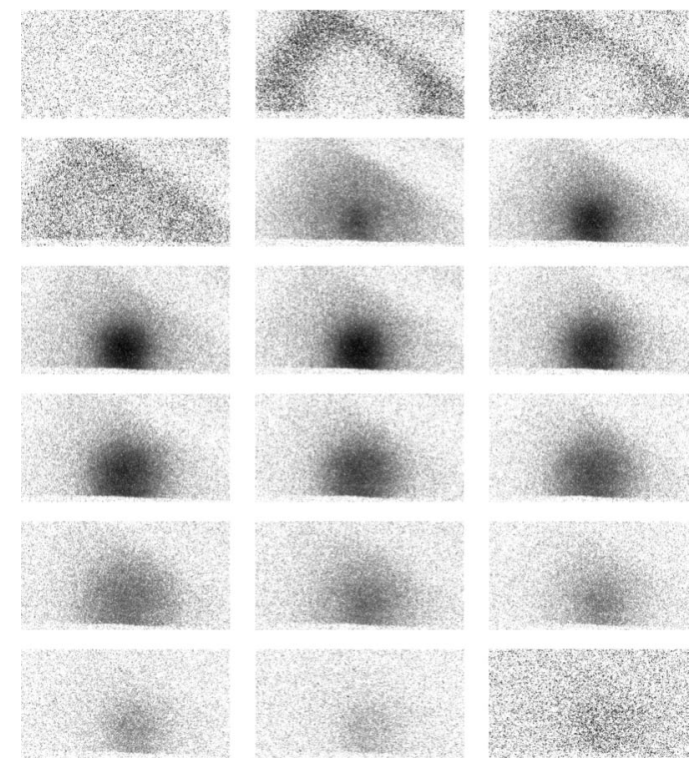

FIG. 4. Pinhole images of the discharge viewed from an on-axis position. First 12 images were taken with 5-ns time steps; for the remaining images time steps of $10 \mathrm{~ns}$ were applied. The first four and the last images were digitally intensified 3.2 times compared to the other ones.

\section{RESULTS}

Inspection of the combined results of pinhole imaging and EUV spectroscopy for the basic version of the source has led to the qualitative identification of three different phases in the main discharge, being an initiation of the pinch with a "ring-shaped" plasma, the pinch itself, and a decay phase with a propagating spherelike plasma "bulb." Quantitative results for each of the phases could subsequently be obtained. Details of the measurement results for each of the phases are described below.

\section{A. "Ring" shape before the pinch}

The first evidence of a ring-shaped plasma before the main pinch was provided by on-axis pinhole images. These are presented in a matrix form in Fig. 4. Each image corresponds to an area of $2.4 \times 1.3 \mathrm{~mm}^{2}$ in the source. The exposed area of each image is limited by the edge of the MCP zone (to the bottom) and a grid line of the filter support (to the top right).

The images are shown in chronological order during the plasma pulse, with time increasing from left to right and from top to bottom. The time step between consecutive images was $5 \mathrm{~ns}$ for the first 12 pictures and $10 \mathrm{~ns}$ for the remaining ones. Since the source was operated in selfbreakdown mode, the definition of an absolute "zero" on the time scale is not straightforward. If the time of maximum pinching - that is, minimal effective radius of the EUV emission-is taken as the zero (between the seventh and eighth image in the series), then the time range between -35 and +85 ns is covered.

The source was operated at a discharge voltage of 13.9 $\mathrm{kV}$, resulting in a pulse energy of $4.6 \mathrm{~J}$. A $50-\mu \mathrm{m}$ pinhole 
was used and for every image, 30 exposures of (effectively) $5 \mathrm{~ns}$ each were integrated, and a dark image from the chargecoupled device was subtracted. The first four pictures and the last one were finally intensified by a factor of 3.2 with respect to the other ones to make the weakest features better visible. Darkest regions correspond to the highest EUV intensities.

The ring phase can be recognized in the second to fourth images, the pinch itself in the subsequent four, and the plasma decay in the remaining images. Note that the limits between the phases, as defined here, are somewhat arbitrary.

From these images it can be derived that the initial phase is relatively short (only about $15 \mathrm{~ns}$ ) compared to the total duration of the discharge (over $100 \mathrm{~ns}$ ). The radiation intensity from this phase of the discharge is weak compared to the pinch radiation. From the images, the inner diameter of the ring at the time of its first appearance in the EUV can be derived to be $1.2 \mathrm{~mm}$; the half-maximum thickness of the ring is about $0.5 \mathrm{~mm}$. The maximum compression velocity is evaluated to be $8 \times 10^{4} \mathrm{~m} \mathrm{~s}^{-1}$.

To identify the EUV lines, and hence the ionization stages, that are responsible for the emission during the initiation phase, spatially resolved spectroscopy was applied to this part of the discharge. As stated already in Sec. II B, the most intense spectral features in this phase are those that are ascribed to the $4 d-4 f$ transitions. The relative intensities of the strongest lines for the ionization stages $8+$ up to $11+$ have been plotted as a function of distance to the axis of symmetry in 5-ns time steps, covering the ring phase and the subsequent compression to the pinch, from the -30 to $0 \mathrm{~ns}$ time marks. The plots are shown in Figs. 5(a) -5 (f) together with the corresponding pinhole images from Fig. 4. Note that, since the spectra and pinhole images were recorded in separate experiments, the correspondence is not perfect, and the pinhole images should only be regarded as a quick reference.

For each time step, the relative populations of the different ionization stages were estimated using Eq. (1). Any possible populations of higher or lower stages than those considered here were simply ignored, and would have led to only a small correction of the relative populations. The result is plotted in Fig. 6. In Fig. 7, the effective average charge derived from these results is plotted versus time, together with the total intensity of the lines under consideration in arbitrary units. Note that the integrated intensity of the considered $4 d-4 f$ lines was already decreasing while the pinch had not yet reached its minimum radius. This point will be discussed in Sec. IV C.

\section{B. Propagating plasma after the pinch}

The third phase of the discharge, the decay after the main pinch, is characterized by a considerable drop of the radiation intensity below the maximum of the discharge and by expansion of the plasma. The expansion appears to have a preferential direction; this can be derived from the off-axis pinhole images as shown in Fig. 8. The source settings were similar to those of the on-axis pictures in Fig. 4. A number of 20-pulse integrated images with $5 \mathrm{~ns}$ effective exposure time were recorded using the $100-\mu \mathrm{m}$ pinhole while varying the delay time in steps of $10 \mathrm{~ns}$. The width and height of each picture correspond to an area of $1.85 \times 1.85 \mathrm{~mm}^{2}$ in the source.

In this figure, the first five or six images correspond to the first two phases of the discharge, the ring and the compression to the pinch. In these two phases, not much EUV radiation is visible from the off-axis position because of shielding of the plasma by the edge of the anode. However, in the subsequent pictures, an apparently nearly spherical "ball" of plasma appears that travels in the vertical direction away from the cathode. Because of the axial symmetry of the source, the plasma must in reality be traveling along the symmetry axis. From this assumption, a propagation velocity can be derived of $3.6 \times 10^{4} \mathrm{~m} \mathrm{~s}^{-1}$. Given that the sonic velocity will be in the order of $1 \times 10^{4} \mathrm{~m} \mathrm{~s}^{-1}$ at most (for temperatures in the $50-\mathrm{eV}$ range) this means that the compression must be supersonic. The propagation velocity compares well in order of magnitude to the average velocity found for the fast ions emitted by the source, as measured by the Faraday cup device.

\section{Comparison between phases}

\section{Relative ionization stage populations}

After the above discussion on qualitative characterization of the different phases during the main discharge, line intensity information from time-resolved spectroscopy can now be studied, giving information on the relative populations of the different ionization stages.

In Fig. 9, line intensities (in arbitrary units) for different stages are plotted against time during the discharge. On the time scale, $t=0$ ns corresponds to the maximum EUV intensity from the plasma. For the EUV spectra, for all ionization stages $4 d-5 p$ lines have been selected for best comparability. However, in the case of Xe XII, next to the (presumed) $5 p$ line at $12.39 \mathrm{~nm}$, also the $4 f$ line at $10.96 \mathrm{~nm}$ is shown for comparison. The line intensity for Xe XIII was calculated by correcting the intensity at $10.80 \mathrm{~nm}$ for the presence of Xe XII radiation: 0.4 times the signal intensity at $10.96 \mathrm{~nm}$ was subtracted from the gross value. This ratio of intensities was found by a parameter study of the spectrum from the discharge.

Note that the Xe XII line array at $12.39 \mathrm{~nm}$ coincides with radiation from Xe VIII. The intensity was not corrected for this contribution, although it is probably dominant at $t$ $\geqslant 80 \mathrm{~ns}$, where the other intensities from the highest ionization stages have nearly dropped to zero.

From the on-axis intensities in Fig. 9, it can be derived that the various ionization stages, ranging from $\mathrm{Xe}^{7+}$ to $\mathrm{Xe}^{11+}$, get populated during a relatively short time interval of about 20 ns. Despite the short time, it can be seen that the ionization stages get populated in order of increasing charge number. The time interval appears to coincide with the ring and compression phases observed in the pinhole camera images. Depopulation of all but the highest ionization stages happens on a much longer time scale. Radiation in the EUV can be observed for about $100 \mathrm{~ns}$ after the moment of maxi- 

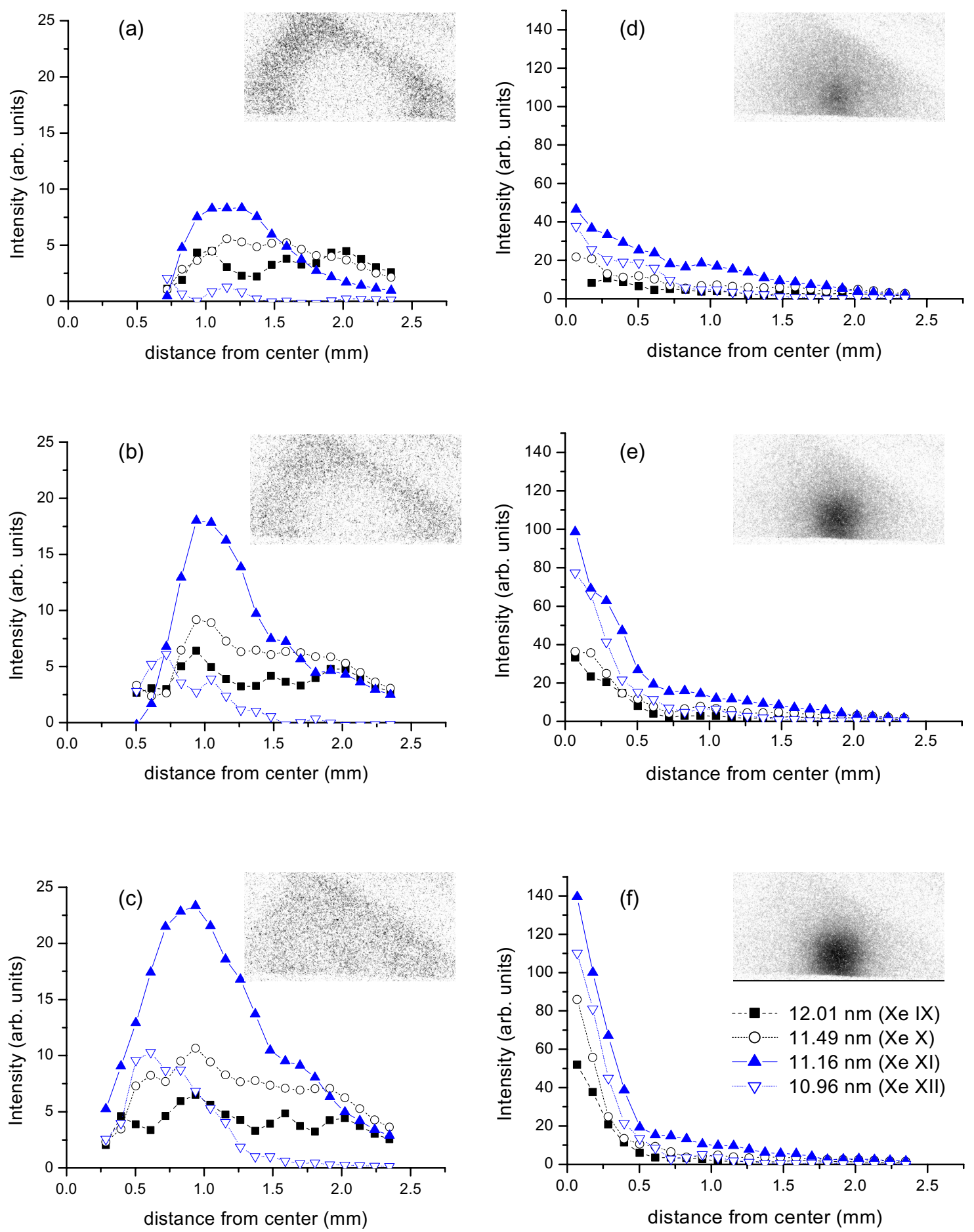

FIG. 5. Measured space-resolved, Abel-inverted relative line intensities of $4 f-4 d$ transitions belonging to different ionization stages of xenon. Different spectra were produced in steps of $5 \mathrm{~ns}$ during the ring phase of the discharge, integrating ten shots for each image. For comparison, on-axis pinhole images are shown in the top right corner of each graph. These are from a different set of experiments, so the correspondence is not perfect.

mum intensities, but in the VUV part of the spectrum between 30 and $90 \mathrm{~nm}$ (not shown), line radiation can be observed for as long as $300 \mathrm{~ns}$ after the maximum intensity was reached.

\section{Relative line strengths within one ionization stage}

Multiple line intensities from one and the same ionization stage do not always show similar time behavior. The clearest case of such dissimilarities in behavior is found for the Xe IX 


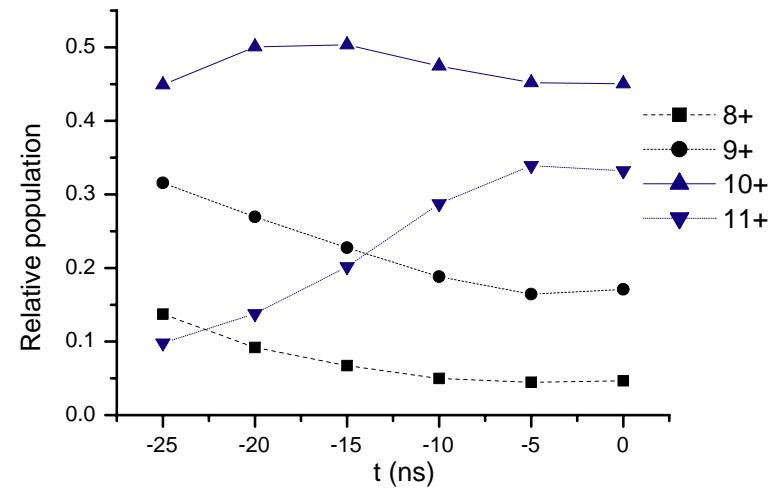

FIG. 6. Estimated relative populations of different ionization stages of xenon as a function of time, in the ring phase of the plasma, and during compression to the moment of minimum pinching radius.

system, and is shown in Fig. 10. When compared to the $4 d-5 p$ lines, the $4 d-4 f$ line shows a relatively larger intensity in the initiation and compression phases of the discharge. The same dissimilarity can be observed in the Xe X spectrum, and, albeit less clearly, in the VIII and XI spectra.

\section{Variation of the discharge voltage}

Due to the self-breakdown operating mode of the source, the only way to change the discharge voltage and the pulse energy was to change the gas flow to the electrodes. As the hollow cathode discharge is operated on the left-hand side of Paschen's curve, an increase of the gas flow (and thereby the background pressure) leads to a decrease of the discharge voltage.

EUV spectra have been recorded at different pulse energies. For each ionization stage that has lines in the EUV, the strongest intensity of the $5 p$ transitions was selected for analysis, except for Xe XIII $(12+)$, of which the $5 p$ transition is overshadowed by other lines, and the top of the $4 f$ array was selected instead. The same procedure as described in Eq. (1) and used in Sec. III A was applied to estimate relative ion stage population densities.

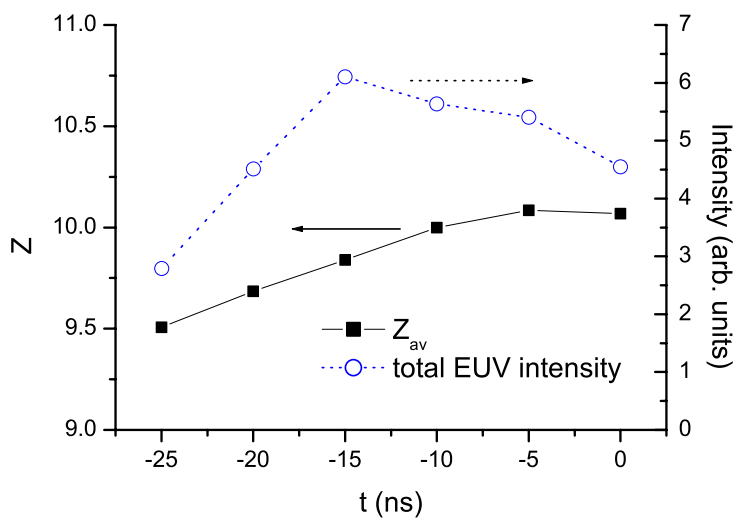

FIG. 7. Total intensity of the considered $4 d-4 f$ EUV lines and effective average charge number as a function of time in the ring phase of the discharge.

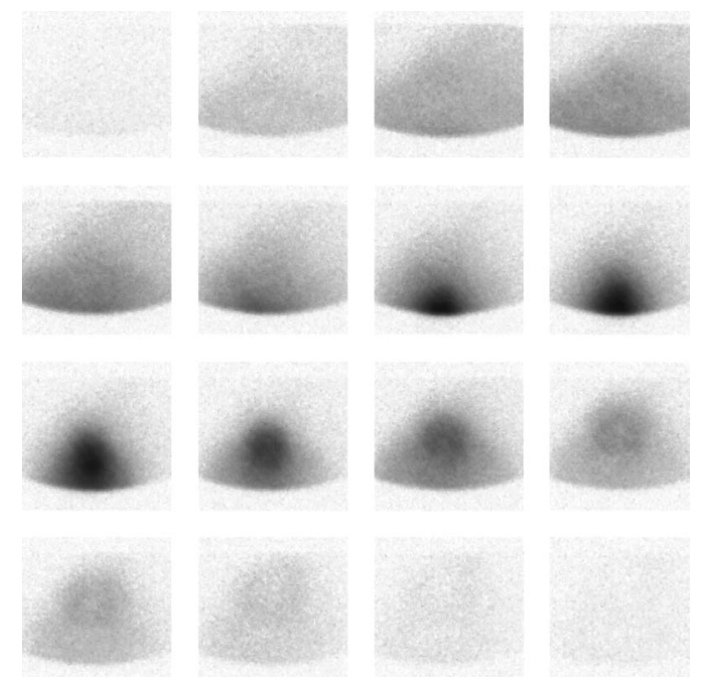

FIG. 8. Shape of the plasma pinch in the EUV as a function of time during the discharge. The MCP gating time was $10 \mathrm{~ns} ; 20$ pulse exposures were integrated per image. The time step between consecutive images is $10 \mathrm{~ns}$. The actual size of each image is $1.85 \times 1.85 \mathrm{~mm}^{2}$.

The results are presented in Fig. 11. The figure shows that the relative populations of the different stages vary relatively slowly with input energy. On the contrary, the total EUV emission shows a sharp, faster than linear increase with pulse energy. The combined effect of a slightly increasing relative population of the $10+$ ionization stage and the behavior of the absolute EUV emission causes a strong increase of the conversion efficiency with pulse energy within the probed regime, up to $4.7 \mathrm{~J}$ input energy.

In time-resolved measurements (not shown here) it was found that the dynamics of the pinch are also influenced by the pulse energy. With increasing energy, the time between the trigger signal and the beginning of the main discharge as well as the time between the appearance of the ring and the moment of minimal pinch radius decreased.

\section{E. Dilution with argon gas}

As mentioned in the Introduction, during an upgrade of the source, the capacitance of the capacitor bank was increased from $48 \mathrm{nF}$ to $674 \mathrm{nF}$, and an adjustment of the electrodes geometry was made including a larger anode hole, enabling a larger collection angle of the EUV radiation from the pinch. In the adjusted setup, a small flow of xenon be-

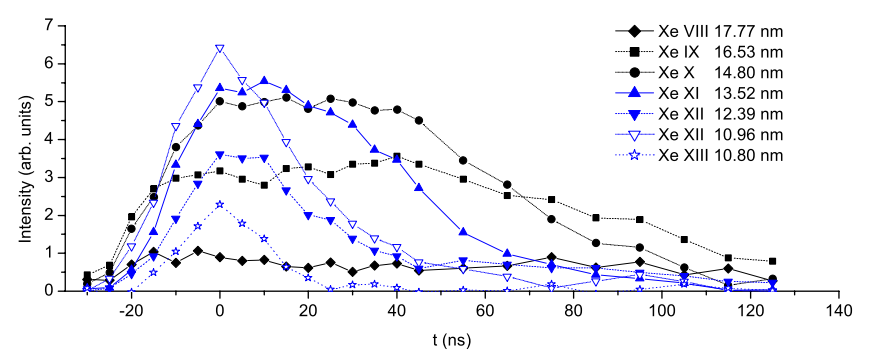

FIG. 9. EUV line intensities vs time measured on-axis at a discharge voltage of $13.9 \mathrm{kV}$. 


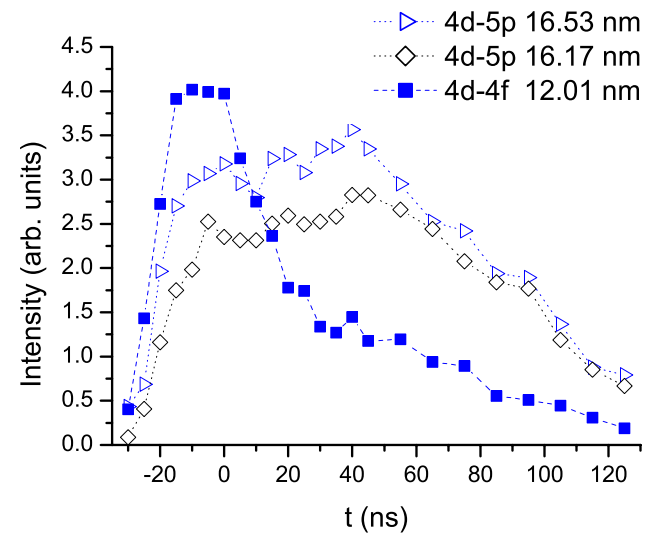

FIG. 10. Different time dependencies of $5 p$ and $4 f$ lines from Xe IX measured on axis.

tween the electrodes was combined with a much larger flow of argon, introduced between the anode and a downstream flow restriction. The application of argon introduced an additional free parameter for adjustment of the plasma properties.

It was found that the conversion efficiency decreased sharply when the xenon flow was reduced from the "standard" value of 8.6 SCCM to a low value of 1.4 SCCM, while at the same time the argon flow was increased from 193 SCCM to 226 SCCM to maintain a constant discharge voltage. The spectra, integrated in time over the discharge pulse, are shown in Figs. 12(a) and 12(b), for high and low xenon flows, respectively.

When both spectra are compared qualitatively, the following appear to be the main differences:

(1) At the higher xenon flow, no Ar lines are visible. At low xenon flow, Ar VIII lines appear in emission.

(2) At low xenon flow, the xenon lines belonging to $4 d-4 f$ transitions are relatively intense compared to the $4 d-5 p$ lines.

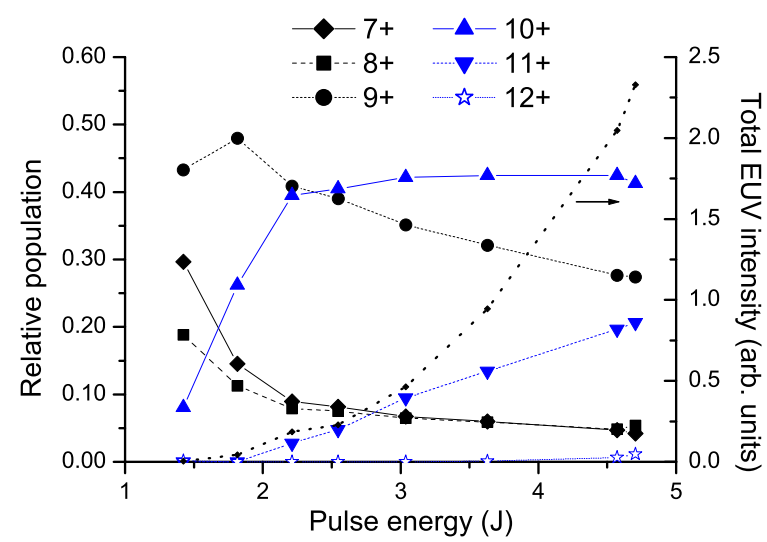

FIG. 11. Effective relative populations of different ionization stages as a function of pulse energy, as derived from line intensities integrated over the duration of the discharge pulse. For explanation of the procedure and used wavelengths, see the main text. The total EUV intensity measured by integrating the intensity of the pulse over the spectrum is also shown.
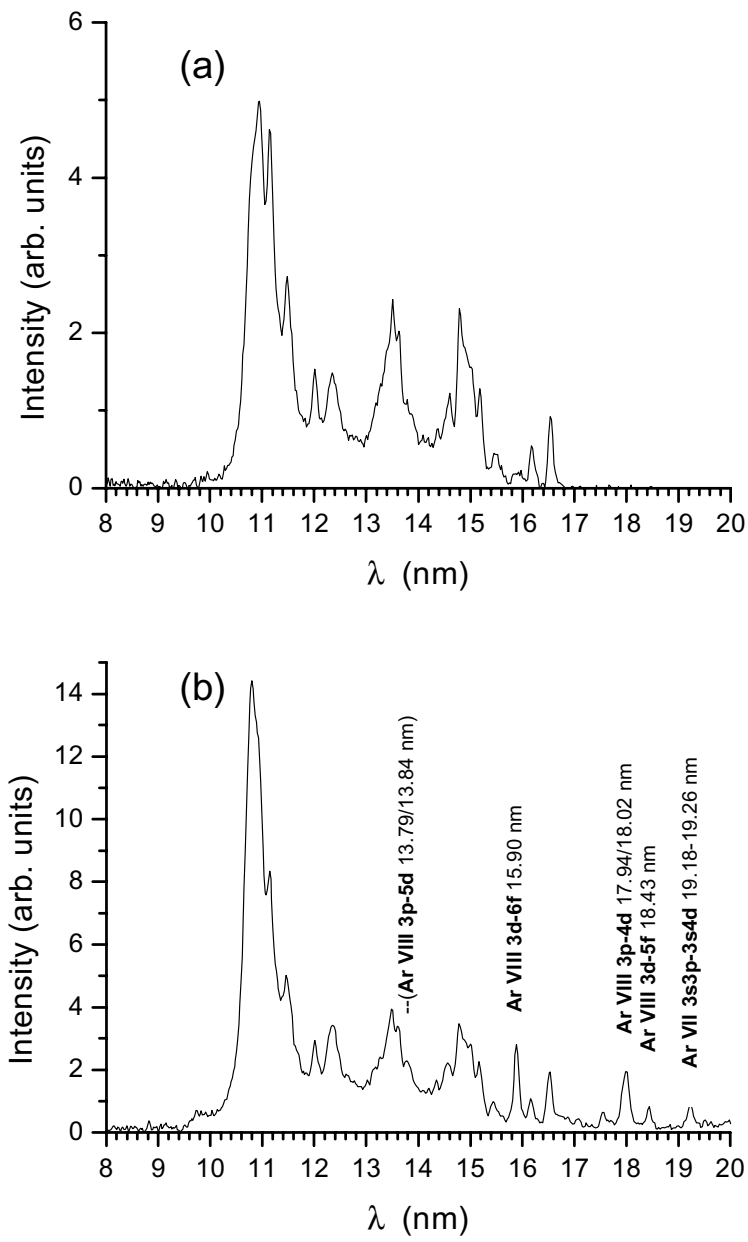

FIG. 12. Time-integrated EUV spectra for pulses with high (a) and low (b) xenon flow into the discharge region. In spectrum (b), EUV lines from argon appear in emission (identification according to Ref. [15]).

(3) From high to low xenon flow, there is a relative increase of the intensity of the Xe XII $5 p$ unresolved transition array (UTA) relative to the intensity of the Xe XI $5 p$ UTA. A similar increase is visible in the Xe XII $4 f$ intensity relative to that of Xe XI. Also, the intensity at $10.80 \mathrm{~nm}$ (tentatively attributed to Xe XIII, since higher stages are unlikely to be present in large quantities) grows relative to that of Xe XII at $10.96 \mathrm{~nm}$. In all cases, radiation from higher ionization stages is favored when the xenon flow is decreased.

\section{DISCUSSION}

\section{A. Origins of the ring shape}

The start of the discharge plasma in the main gap in a ring shape was an unexpected effect observed by pinhole imaging. In case the pulse would start as a diffuse discharge with a flat current distribution, the Lorentz force on a moving charged particle would scale linearly with the distance form the axis of symmetry. From this, it can be derived that the current distribution within the compressing plasma would remain flat (within a decreasing radius). However, any initial inhomogeneity in the distribution could grow due to a corresponding deviation in the Lorentz force. 
Now, the question is what could be the cause of the initial inhomogeneity in the current distribution. One factor is the cathode geometry: The generation of secondary electrons by ions on the cathode surface during the high-current phase will take place mainly on the cathode edges, which have a ring shape, causing also a ring-shaped distribution of the current in the plasma phase.

Second, the skin effect, i.e., the exclusion of the fastvarying current from the center of the plasma, might also cause the current to run mainly along the edges of the plasma at the start of the high-current phase.

\section{B. Preferential direction of plasma expansion}

In the pinhole images of Fig. 8, it was seen that the pinch plasma does not decay simply isotropically, but it has a preferential direction along the axis. In the present results, only expansion in the forward direction is visible. However, the most likely explanation of the anisotropy is the anisotropy in the Lorentz force. While the pinch plasma is confined in the radial direction by the Lorentz force, it is free to expand in the axial direction. Therefore, a similar expansion in the inward direction (i.e., towards the cathode hole) is expected. A second contribution may come from the "zipper effect," in which the plasma is accelerated in an axial direction due to an axial component in the Lorentz force.

\section{Causes of spectral changes over time}

In this section the main differences between the xenon EUV spectra from different phases of the discharge will be discussed. In the discussion, the focus will be on the relative populations of the different stages, and for radiation from a single stage, mainly on the lines emitted by Xe IX, since these lines (and their transition probabilities) are well established in literature, and also resolvable individually (instead of only in line arrays). The intensities of the main Xe IX EUV lines versus time during the discharge were plotted in Fig. 10.

All of the identified transitions in the EUV are resonant, i.e., the lower level is (very close to) the ground state of the ion. This means that the wavelength of the line is a direct measure of the energy of the upper level. Therefore, the intensity of the lower wavelength lines (the $4 d-4 f$ transitions) relative to that of the larger wavelength $(4 d-5 p)$ lines, is an indication of the electron temperature of the plasma, assuming a given excitation/deexcitation balance.

From rough estimates of the ion density (up to $10^{24} \mathrm{~m}^{-3}$ ), average ionization stage $(\sim 10)$, and electron temperature (up to $\sim 35 \mathrm{eV}$ ) in the pinching plasma, it can be deduced that the excitation/deexcitation balance in most phases of the plasma will be neither pure corona nor pure LTE, but rather in between the two. In case of LTE, and an optically thin plasma, the intensities of the individual lines will be proportional to their transition probabilities. On the other hand, the intensities have an extra $1 /(\Delta E)^{3}$ dependency in pure corona balance compared to LTE, since in this case the intensity of the radiation is proportional not to the optical transition probability but to the electronic excitation rate from the ground state. This means that with similar electron temperature, a less dense plasma will have relatively stronger radiation at larger wavelengths compared to a more dense one.

During the ring phase of the plasma, the $4 f$ intensities are relatively high, in spite of the necessarily low density of the plasma in this phase. This is an indication of a high electron temperature in this phase.

During compression of the ring to the actual pinch, the $4 f$-line intensities remain high and that of the $5 p$ lines increase. The increase of the intensity of the $5 p$ lines can easily be explained by an increase of the density of the plasma (and resulting shift to a balance that is closer to LTE). The fact that the total intensity of the $4 f$ lines does not increase correspondingly shows that the electron temperature must already be dropping at this time. This assumption is supported by the slight decrease of average ionization stage shown by detailed EUV spectroscopy in the ring phase (Fig. 7).

Admittedly, similar trends would be observed if, in this phase, the plasma would become optically thick to some of the $4 f$ lines. However, neither the details of the experimentally determined EUV spectra, nor comparison with the Planck limit derived from calculated transition probabilities and the above plasma parameters point in this direction.

During the decay of the pinch, the $4 f$ intensity decreases quickly, while in the case of Xe IX the $5 p$ line intensity stays almost constant for some time (about $40 \mathrm{~ns}$ ). The relatively fast decrease of the $4 f$ line intensity can be explained by cooling of the plasma and a shift of the population balance back towards Corona. For the $5 p$ lines, the cooling is compensated by repopulation of the $\mathrm{Xe}^{8+}$ ionization stage by recombination from higher stages.

Even with a low electron temperature, the relatively low intensity of the $4 f$ line is remarkable. Also, it may be noted that after $t=60 \mathrm{~ns}$, both $5 p$ intensities become nearly equal, while there was a clear difference in the intensities earlier in the discharge. As the upper energy levels for both lines are very close together, such a change cannot be explained by a change in the plasma parameters alone. A possible cause for the change is a "capture radiative cascade" effect, where recombination into an excited level of $\mathrm{Xe}^{8+}$ and subsequent radiative decay to lower levels leads to a spectrum that is different from either normal LTE or corona balance.

Summarizing, it is stated that in the initial (ring) phase, the excitation/deexcitation balance is between pure LTE and corona balances, and the electron temperature is high; during the pinching, the density increases and the excitation balance moves closer to LTE, but electron temperature drops; and during the plasma decay, temperature drops further and the balance moves back towards corona. In the latter phase, specific recombination effects might have an influence on the shape of the spectrum.

\section{Absence of Ar IX lines and changes in the Xe spectrum at low Xe flow}

When the source was operated with the argon buffer gas at low xenon flow, some argon lines became visible. All these can be identified as belonging to the Ar VIII spectrum [15]. However, comparison of ionization energies of the most 
abundant xenon ions $(180 \mathrm{eV}, 205 \mathrm{eV}, 231 \mathrm{eV}$, and $258 \mathrm{eV}$ for $8+$ up to $11+[6])$ with those of $\mathrm{Ar}^{7+}$ and $\mathrm{Ar}^{8+}(143 \mathrm{eV}$ and $422 \mathrm{eV}$ [16]) shows that in the pinch, almost exclusively, eight times ionized argon should be present: $\mathrm{Ar}^{7+}$ has such low ionization energy that it should be completely ionized through to the next stage, whereas that of $\mathrm{Ar}^{8+}$ is so high that $\mathrm{Ar}^{9+}$ will not be populated considerably. Also, it is known that $\mathrm{Ar}^{8+}$ has some lines in the EUV range of 8-20 $\mathrm{nm}$ [15]. However, the upper states responsible for these lines all have energies in excess of $300 \mathrm{eV}$ above the ground state of this ion, and therefore will hardly be populated in the pinch plasma with a temperature of "only" about $35 \mathrm{eV}$. This explains their absence in the relatively cool pinch plasma.

The difficulty to either further ionize or excite the $\mathrm{Ar}^{8+}$ ions present in the plasma may be responsible for an increase of the electron temperature compared to the discharge with larger xenon flow. The electrons simply cannot lose their energy easily in either excitation or ionization. The increase in electron temperature is the most likely cause of the shift towards higher ionization stages observed in the xenon contribution to the spectrum. It may also be a large contributor to the shift towards relatively more intense radiation from the short-wavelength $4 f$ radiation compared to the $5 p$ transitions in xenon.

It should be noted that there might be a second contributor to this shift, which is the optical density of the plasma. Even though there are no indications in the present experimental results that the radiation from the plasma is optically thick at any time during the discharge, it could play a role: it is possible due to the dilution with argon that at low xenon flow, the $4 f$ transitions are less optically dense than at high xenon flow. Hence they might be enhanced compared to the $5 p$ transitions of the same stages.

\section{CONCLUSIONS}

The experimental results presented in this paper may serve as proof of the good applicability of the cost-efficient combination of fast-triggerable MCP and photocamera diagnostics for characterization of EUV sources by pinhole imaging and EUV spectroscopy.

The described experiments have led to improved understanding of the pinch dynamics of the Philips hollow cathode discharge. A plasma was observed inside the cathode, it was shown that high ionization stages are present already before the main pinch occurs, and it was observed that the initiation of the main discharge occurs as a ring-shaped plasma.

In the EUV band from 9 to $18 \mathrm{~nm}$, contributions from many ionization stages of xenon (ranging from $7+$ to $12+$ ) can be distinguished, and their relative populations during the plasma pulse can in principle, be monitored.

The behavior of individual line intensities, especially for the Xe IX spectrum, can give further insight in the characteristics of the plasma. Already, they provide indications of shifts in temperature and the excitation/deexcitation balance during the discharge. It was argued that they indicate a relatively high electron temperature and a balance between LTE and corona during the ring phase of the discharge, and lower electron temperature and the possible presence of radiative cascade processes during the decay of the plasma.
[1] V. Banine and J. Moors, Proc. SPIE 4343, 203 (2001).

[2] V. Banine, O. Frijns, R. Moors, R. Gontin, H. Franken, K. Koshelev, E. Kieft, K. Garloff, and Yu. Sidelnikov, Proceedings of the first International EUVL Symposium, International SEMATECH, Dallas, Texas, 2002 (unpublished).

[3] K. Bergmann, O. Rosier, R. Lebert, W. Neff, and R. Poprawe, Microelectron. Eng. 57-58, 71 (2001).

[4] K. Bergmann, O. Rosier, W. Neff, and R. Lebert, Appl. Opt. 39, 3833 (2000).

[5] K. Bergmann, G. Schriever, O. Rosier, M. Müller, W. Neff, and R. Lebert, Appl. Opt. 38, 5413 (1999).

[6] R.D. Cowan, The Theory of Atomic Structure and Spectra (University of California Press, Berkeley, 1981) (code in a version for personal computers, edited by A. Kramida, at http://das101.isan.troitsk.ru/cowan.htm).

[7] For product information see http://www.schulz-si.com.

[8] C. Martin and S. Bowyer, Appl. Opt. 21, 4206 (1982).
[9] S.S. Churilov and Y.N. Joshi, Phys. Scr. 65, 40 (2002).

[10] V. Kaufman and J. Sugar, J. Opt. Soc. Am. B 1, 38 (1984).

[11] M.A. Klosner and W.T. Silfvast, J. Opt. Soc. Am. B 17, 1279 (2000).

[12] W. Benker, J. Christiansen, K. Frank, H. Gundel, W. Hartmann, T. Redel, and M. Stetter, IEEE Trans. Plasma Sci. 17, 754 (1989).

[13] J.-P. Boeuf and L.C. Pitchford, IEEE Trans. Plasma Sci. 19, 286 (1991).

[14] R. Stuik, H. Fledderus, P. Hegeman, J. Jonkers, M. Visser, V. Banine, and F. Bijkerk, in Proceedings of the Second SEMATECH Workshop on Extreme UV Lithography, San Francisco, 2002 (unpublished).

[15] B.C. Fawcett, A. Ridgeley, and G.E. Bromage, Phys. Scr. 18, 315 (1978).

[16] CRC Handbook of Chemistry and Physics, 72 ed., edited by D. R. Lide (CRC Press, Boca Raton, FL, 1991). 